\section{Use of social media by health professionals in South Africa}

To the Editor: The article by Dr Kubheka ${ }^{[1]}$ contains an important error. I did not advise a 'breast-feeding mother ... to wean her child on a low-carbohydrate and high-fat diet'. I gave generic nutritional information to a (presumed) mother who asked a generic 'we' question on behalf of 'moms' and 'babies'. In the course of the 26-day multimillion-rand Health Professions Council of South Africa (HPCSA) hearing into my professional conduct, it was never established that the woman was in fact a mother, or that she was herself breastfeeding. Nor did she lay the complaint (of unprofessional conduct) against me, but this is perhaps beside the point.

What is more important is that the HPCSA based their charge against me on the presumption that by answering a single tweet, whether or not that tweet sought generic information or specific medical advice, I (and therefore all medical practitioners doing the same in the future) had intentionally entered a doctor-patient relationship with the person posting the tweet. Kubheka's article specifically distinguishes between 'generic' and 'direct medical' advice. Hence her assertion that 'it is advisable that professionals share generic information online, and avoid responding with direct medical advice to individuals. This distinction is the difference between responding to an 'I' or a 'we' question, and the question I answered was a 'we' question seeking generic medical information.

The HPCSA's Professional Conduct Committee judging my case concluded that answering a tweet does not, by itself, initiate a doctorpatient relationship. My legal team argued inter alia that both parties must first agree before there is a doctor-patient relationship, as had clearly not happened in this case.

Any doctor willfully attempting to enter a doctor-patient relationship on Twitter must very likely act unprofessionally, since there is a high probability that the act of 'treating' a patient on Twitter will involve supersession. This is because patients resorting to Twitter are not actually seeking medical care, so they have no reason to address any requests to their personal caregivers.

This is why Kubheka's advice that 'any medical discussion professionals enter into on social platforms be accompanied by the advice that patients must consult their practitioners' quite misses the point. Twitter is an information-sharing platform that cannot be equated with what happens in the doctor's consulting room. Importantly, persons access Twitter to seek information that will help to guide their decision on what medical care they might seek from the medical profession in the future. They understand that Twitter is not a medium for the provision of medical care.

Logically, persons seeking information on Twitter do so for reasons either of (no) cost and convenience, or specifically because they have not been able to source that information elsewhere. Had they already been satisfied with information received from caregivers, they would not have resorted to Twitter. This is a fact of life that legislation or guidelines, however framed, will never eradicate.

The second reason why it is not possible to provide ethical medical care via Twitter is that any registered medical practitioner wishing to do so must request the patient to provide personal medical information. Exposing such information publicly on a social media platform could constitute a breach of medical ethics. Kubheka's warning here is important: 'Crucially, information shared online is in the public domain and has relative permanence.'
One conclusion might be that the existing regulations for professional behavior by medical professionals adequately prescribe the boundaries of ethical conduct on Twitter.

What became very apparent during my HPCSA 'hearing' was the widespread ignorance within the profession of exactly how the general public use Twitter, specifically (but also other forms of social media), to access medical advice and information. There are four relevant points here:

Firstly, as I have described, patients do not seek medical care on Twitter, and so they will not consult their personal physicians using this medium. Rather, they seek the opinions of those doctors, scientists and lay persons whose hobbies include spending time sharing such information. Few clinicians with busy medical practices will have sufficient leisure time to also be active on Twitter.

Secondly, the vast majority of medical questions on Twitter that I encounter do not seek specific medical advice, such as in asking 'Should I take a statin drug if my blood cholesterol is elevated?' Much more likely are questions of clarification, for example, 'What medical benefits can a patient expect if she uses a statin?' Experienced medical hobbyists providing information on Twitter would, as Kubheka suggests, likely avoid answering the first question directly. Rather, they would more probably provide information about the risks and benefits of these drugs. So apprised, the patient is better able to make an informed decision about what form of medical therapy is likely to be best.

When patients do seek medical care, they do so explicitly by asking for a medical appointment, indicating that they wish to establish a conventional doctor-patient relationship following the conventional channels.

Thirdly, Twitter is almost instantly self-correcting. Incorrect advice posted on Twitter will almost immediately be challenged by those whose experience or expertise suggests the information in a tweet to be incorrect. This is a key value of this very public medium of information sharing

My HPCSA case provided an excellent example of this. Within minutes of my disputed tweet going live, two dietitians had expressed the opinion that my advice was wrong, and had offered alternative options. The person who had posted the original tweet now had a range of options to consider, as is the desirable outcome in this public form of information sharing.

Fourthly, the use of social media has democratised the delivery of medical information, a critical point to which medical professionals will need to adapt if they wish to attract and retain social-mediasavvy patients. Importantly, social media publicises in a very open way those therapeutic interventions that either do or do not work. Essentially, there is no place for failed therapies or charlatan activities to hide on social media.

Naturally, this is challenging for those taught to believe that registered medical practitioners are the sole source of credible medical information and advice, provided only as part of a conventional doctor-patient consultation.

\section{T D Noakes}

Retired. The Noakes Foundation, Bergvliet, Cape Town, South Africa Noakes@iafrica.com @ProfTimNoakes

1. Kubheka B. Ethical and legal perspectives on use of social media by health professionals in South Africa. S Afr Med J 2017;107(5):386-389. https://doi.org/10.7196/SAMJ.2017.v107i5.12047 